\title{
Evolving the Web into a Global Data Space
}

\author{
Christian Bizer \\ Freie Universität Berlin \\ Germany \\ chris@bizer.de
}

\begin{abstract}
Linked Data technologies provide for setting links between records in distinct databases and thus to connect these databases into a global data space. Over the last years, Linked Data technologies have been adopted by an increasing number of data providers, including the US and UK governments as well as mayor players in the media and pharmaceutical industry, leading to the creation of a global Web of Data. In his talk, Prof. Christian Bizer will give an overview of the Web of Data as well as the architecture of Linked Data applications that work on top of this data space. Afterwards, he will discuss how the openness and self-descriptiveness of Linked Data provide for splitting data integration costs between data publishers, data consumers and third parties and thus might enable global-scale data integration in an evolutionary, pay-as-yougo fashion. He will close with an overview of the research challenges that the Linked Data community currently faces.
\end{abstract}

